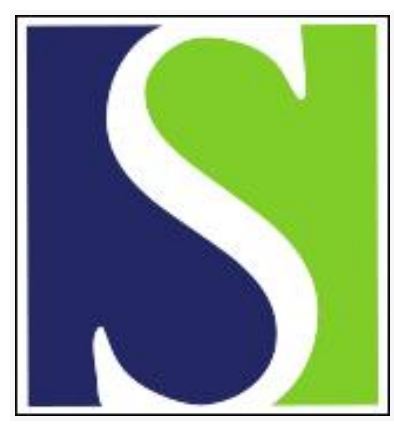

Scand J Work Environ Health 2000;26(2):87-92

https://doi.org/10.5271/sjweh.516

Issue date: Apr 2000

Human brain activity during exposure to radiofrequency fields emitted by cellular phones

by Hietanen M, Kovala T, Hämäläinen A-M

Key terms: electroencephalogram; electromagnetic fields; exposure; mobile phone; nonionizing radiation

This article in PubMed: www.ncbi.nlm.nih.gov/pubmed/10817372

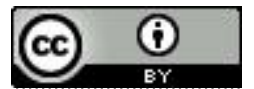




\title{
Human brain activity during exposure to radiofrequency fields emitted by cellular phones
}

\author{
by Maila Hietanen, PhD, ${ }^{1}$ Tero Kovala, MD, ${ }^{2}$ Anna-Maija Hämäläinen, $M S C^{\dagger}$
}

\begin{abstract}
Hietanen $M$, Kovala T, Hämäläinen A-M. Human brain activity during exposure to radiofrequency fields emitted by cellular phones. Scand J Work Environ Health 2000;26(2):87-92.

Objectives The aim of this study was to explore the possible influence of radiofrequency (RF) radiation exposure on human brain function.

Methods The electroencephalographic (EEG) activity of 19 volunteers was quantitatively analyzed. Ten of the subjects were men ( $28-48$ years of age) and 9 were women ( $32-57$ years of age). The sources of exposure were 5 different cellular phones (analogue and digital models) operating at a frequency of $900 \mathrm{MHz}$ or $1800 \mathrm{MHz}$. The EEG activity was recorded in an awake, closed-eyes situation. Six 30-minute experiments, including 1 sham exposure, were made for each subject. The duration of a real exposure phase was 20 minutes.

Results Exposure to one of the phones caused a statistically significant change in the absolute power at the delta band of the EEG recording. However, no difference was seen in the relative power of the same band, and no changes occurred during exposure to other phones at any frequency bands.

Conclusions The findings of this study suggest that exposure to radiofrequency fields emitted by cellular phones has no abnormal effects on human EEG activity. The observed difference in 1 parameter was probably caused by statistical chance.
\end{abstract}

Key terms electroencephalogram, electromagnetic fields, exposure, mobile phones, nonionizing radiation.

In recent years, the use of handheld mobile phones, or cellular phones as they are generally called, has increased drastically in modern societies. Along with their rapid expansion, suspicions about possible adverse consequences from exposure to radiofrequency (RF) fields emitted by the antennas have been a subject of considerable interest among both the scientific community and the general public.

During normal use, cellular phones are held in close proximity to the head, which is therefore exposed to relative high RF fields in comparison with the rest of the body. The heating of biological tissues through the absorption of RF energy has been well known for decades. The RF power emitted by cellular phones is, however, very low, and, according to dosimetric studies, no significant warming of biological tissue occurs (1-3). Consequently, within RF bioeffects research, much effort is focused on the possible nonthermal effects on the central nervous system, in particular on the brain.

Evidence of an influence of low frequency (50-60 $\mathrm{Hz}$ ) electromagnetic fields on electroencephalographic (EEG) activity has been provided by animal and human studies (4-7). Thus far, only a few studies have concerned the possible influence of the cellular phones now in use on human EEG activity. In a single-blind study by Reiser and his colleagues (8), the RF exposure sources were a digital mobile phone $(900 \mathrm{MHz})$ and a medical therapy instrument $(150 \mathrm{kHz})$. The therapy device was situated at a distance of $3-5 \mathrm{~cm}$ from the test person's skin, whereas the cellular phone was placed as far as 40 $\mathrm{cm}$ from the subject's head. Both devices caused an increase in the frequency bands alpha $a_{2}$, beta ${ }_{1}$ and beta $a_{2}$, with a delay of approximately 15 minutes after the cellular phone exposure.

The effects of short cellular phone exposure on the awake EEG activity of young ( $21-35$ years) men under closed-eyes conditions have been studied by Röschke \& Mann (9). No statistical differences in the spectral power densities at various frequency bands were observed between real and sham exposure situations. In a recent study by Freude and her colleagues (10), the effect of the electromagnetic fields emitted by cellular phones on the brain activity of young ( $21-29$ years) male volunteers was studied in connection with experimental tasks.

1 Finnish Institute of Occupational Health, Helsinki, Finland.

2 Helsinki University Hospital, Laboratory of Diagnostics, Division of Clinical Neurophysiology, Helsinki, Finland.

Reprint requests to: Dr Maila Hietanen, Finnish Institute of Occupational Health, Topeliuksenkatu 41 a A, FIN-00250 Helsinki, Finland. [E-mail: maila.hietanen@occuphealth.fi] 
A significant decrease in the slow-brain potentials was observed in the central and temporo-parieto-occipital brain regions.

In our study, we assessed abnormal changes in human EEG activity during the use of ordinary cellular phones. The objective was to evaluate whether exposure to RF fields emitted by the antennas disturbs the electric functions of the human brain.

\section{Subjects and methods}

\section{Subjects}

Nineteen healthy volunteers were included in our study. Of the test subjects, 9 were women [mean age 46.6 (range $32-57$, SD 9.0 years], and 10 were men [mean age 37.7 (range 28-48, SD 6.6) years]. No EEG-affecting medication was used by any test person. The subjects were recruited by a personal phone call from the reference study group for normal EEG activity at the Finnish Institute of Occupational Health. Thus the subjects were known to have normal EEG activity, and all of them were familiar with the EEG recording. The volunteers were informed in detail about the purpose and procedures of the study, and a written consent and health information inquiry form that had been filled out by the volunteers was obtained. The study design was in accordance with regulations for the protection of research subjects, approved by the Ethical Committee of the Finnish Institute of Occupational Health.

\section{Exposure sources}

Five different types of analogue and digital handheld cellular phones were used as the exposure sources. Three of them were analogue $900 \mathrm{MHz}$ NMT (Nordic mobile telephone) phones, which have been widely used in the Nordic countries since 1982. The NMT phones were

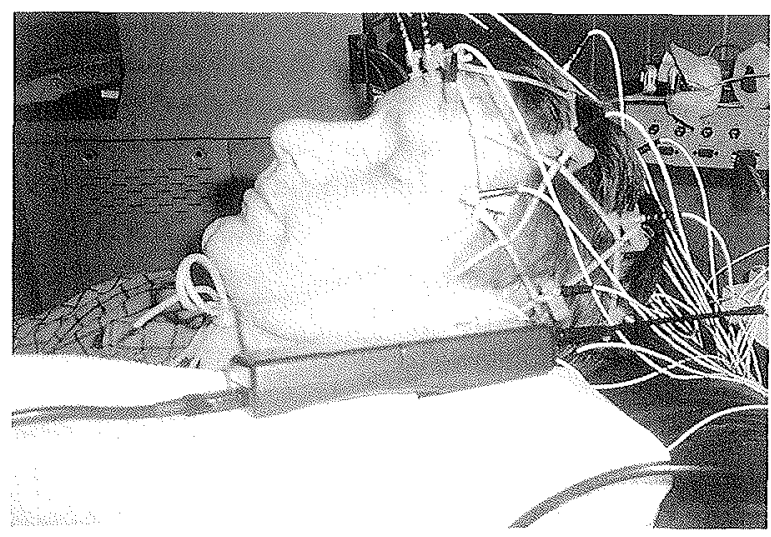

Figure 1. Cellular phone exposure set-up during the electroencephalographic recordings. produced by 3 manufacturers, and each of them had a different antenna, either a helix, fixed whip, or extended whip antenna. In addition to the NMT phones, we used a digital GSM (global system for cellular communications) phone, and a digital PCN (personal communication network) phone. The GSM phones, widely used in Europe and in the Asian countries, operate at a frequency of 900 $\mathrm{MHz}$, while the PCN system utilizes the frequency band of $1800 \mathrm{MHz}$. The range of the peak output power of the cellular phones was $1-2 \mathrm{~W}$.

\section{EEG recording}

EEG recording is a useful tool for investigating rapid physiological phenomena of the brain. The changes in the electrical activity of the brain are described as EEG waveforms and are measured by the potential difference between 2 electrodes. The recordings were performed with Cadwell Spectrum 32 equipment with 21 channels. Unpolarizable surface electrodes were attached to the scalp according to the international $10-20$ system. Two additional electrodes were used: an Fpz electrode (between the Fp1 and Fp2 electrodes) and an $\mathrm{Oz}$ electrode (between the $\mathrm{O} 1$ and $\mathrm{O} 2$ electrodes). The ground electrode was positioned between the $\mathrm{C} 3$ and $\mathrm{T} 3$ electrodes. The electrode impedances were kept below $5 \mathrm{k} \Omega$.

The EEG recording apparatus used in our study was well protected against external interference. The EEG wires were bunched together to avoid the induction of external fields to the wires. The material of the amplifier box was aluminum, and, therefore, it acted as a Faraday cage. The voltage in the cable from the amplifier to the EEG device was a few volts, and the cable was not sensitive to external fields. Immunity to interference was tested by using saline water in a basin and a cardboard plate. The EEG electrodes were put into water through the cardboard, containing holes to keep the electrodes aloof to simulate their location on the head. Each cellular phone was moved close to the electrode wires without touching them, and the EEG signal was evaluated.

For filtering the signal, a 70-Hz low-pass filter and a $50-\mathrm{Hz}$ Notch filter were used. The sampling frequency was $205 \mathrm{~Hz}$, and a 12-bit analogue-digital converter was used. The recorded EEG signals were stored on the optical disc for later analysis. The visual interpretation, choice of epochs, and quantitative analyses were performed off-line with a Cadwell RDC-32 reading station. The selection of the epochs started as the cellular phone was emitting for 10 minutes. Altogether 24 epochs, each lasting 2.5 seconds, were gathered (totally 1 minute). The epochs chosen had to be artifact-free, without signs of a lowered level of vigilance during the recording.

\section{Experimental procedure}

Six EEG recordings, one of which was a 30-minute sham exposure, were taken for each subject. The 5 exposure 
trials included 20 minutes of RF field exposure and 10 minutes of sham exposure ( 5 minutes at the beginning and 5 minutes at the end). The order of different exposure conditions was randomized. The EEG recordings were performed in a silent, half-darkened room at the laboratory of clinical neurophysiology of the Finnish Institute of Occupational Health. The subjects were sitting in a comfortable chair, keeping their eyes closed, but staying awake. Only 1 test per day was done for each subject.

The position of the cellular phone simulated the typical distance of a mobile phone from the head, with the exception that the test persons did not hold the phone by hand. It was located alongside a test person's head at a distance of 1.0 (SD 0.5) $\mathrm{cm}$ from the head (figure 1). The phone was adjusted with a cushion, except for one, which needed a stand because of its structure. The antennas were configured to transmit at maximum power during the tests. The functioning of the phones was checked with a power meter after being switched on and occasionally during the EEG recordings. To keep the test situation blind for the subjects (eg, to keep the test persons

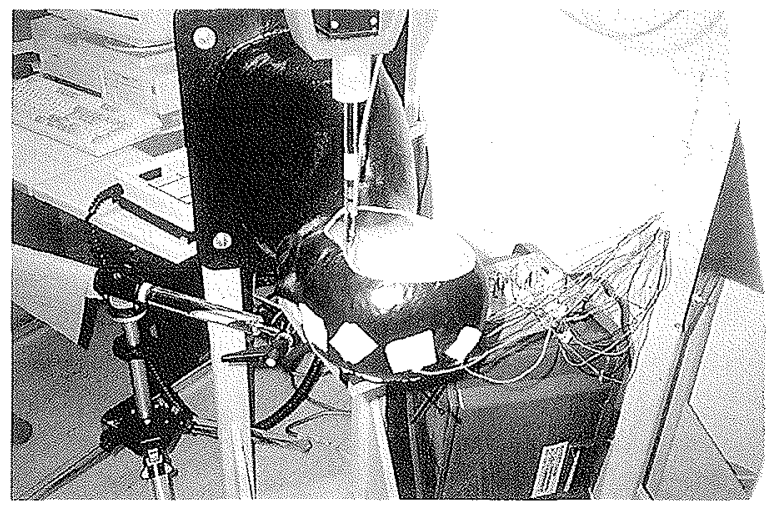

Figure 2. Scanning system for measurements of specific absorption rate with a phantom head.

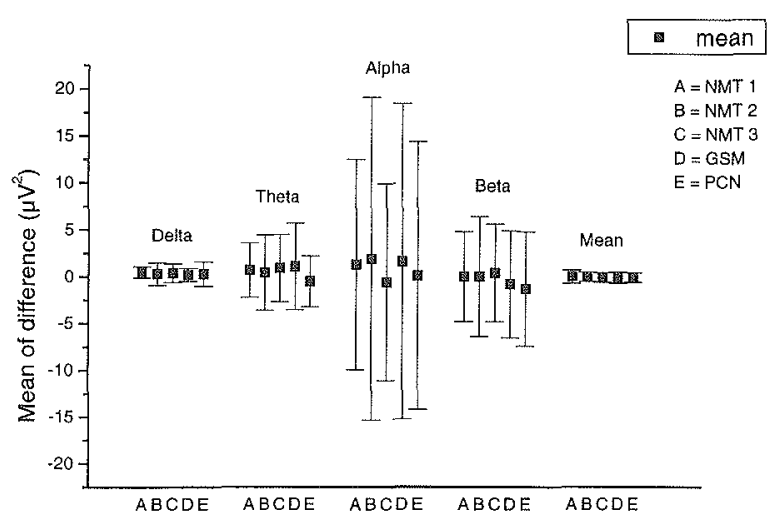

Figure 3. Mean difference in the absolute powers in the centroparietal region with 5 different phone models. unaware of whether the telephone was on or off) the phones were operated via a computer without auditory transmission.

The vigilance of the subjects was controlled during the EEG recordings by inspecting their eye movements and EEG activity at various frequency bands over the

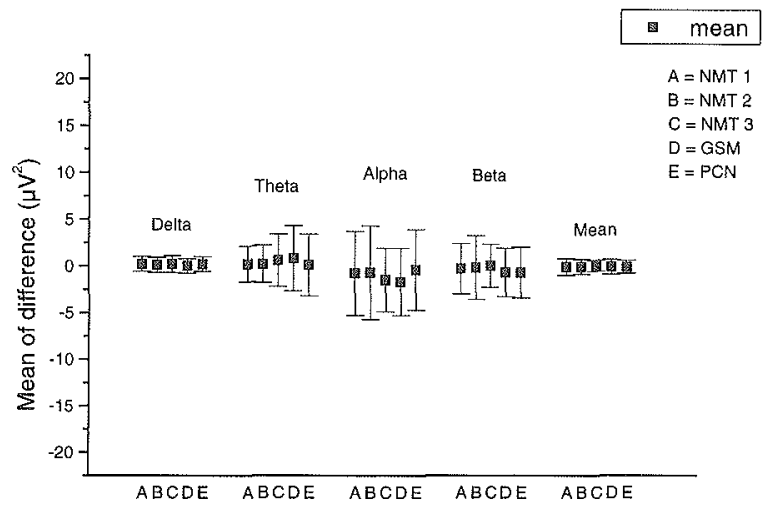

Figure 4. Mean difference in the absolute powers in the frontal region with 5 different phone models.

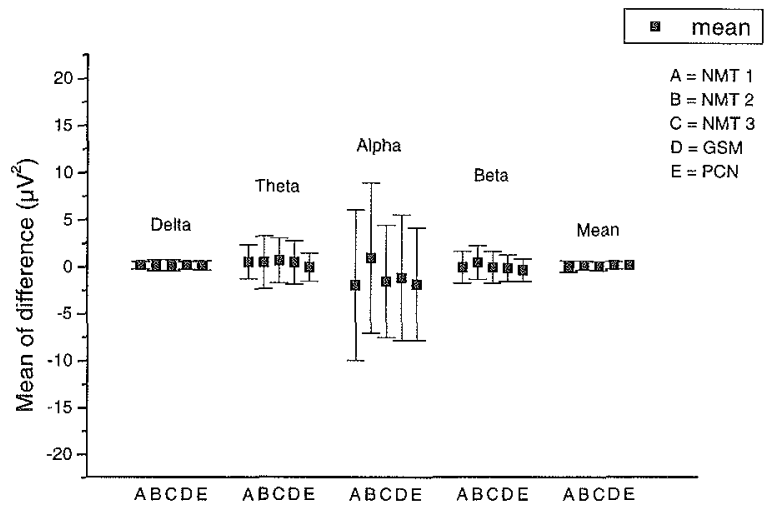

Figure 5. Mean difference in the absolute powers in the temporal region with 5 different phone models.

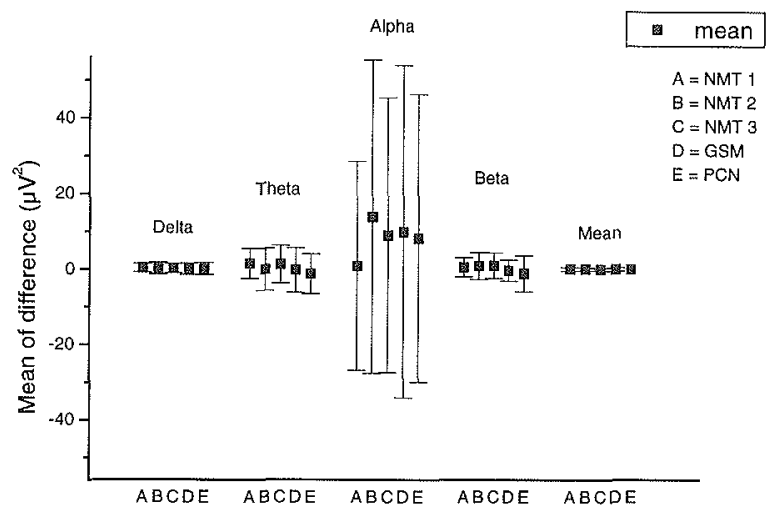

Figure 6. Mean difference in the absolute powers in the occipital region with 5 different phone models.

Scand J Work Environ Health 2000, vol 26, no 2 
Table 1. Means, standard deviations, 95\% confidence intervals, and P-values of the difference between real and sham exposure for Nordic mobile telephone)

\begin{tabular}{|c|c|c|c|c|c|c|c|c|c|c|c|c|c|c|c|c|}
\hline \multirow[t]{4}{*}{ Region } & & & & & & & & & & & & & \multicolumn{4}{|c|}{ EEG parameter } \\
\hline & \multicolumn{16}{|c|}{ Absolute power $\left(\mu V^{2}\right)$} \\
\hline & \multicolumn{4}{|c|}{ Delta } & \multicolumn{4}{|c|}{ Theta } & \multicolumn{4}{|c|}{ Alpha } & \multicolumn{4}{|c|}{ Beta } \\
\hline & Mean $^{\mathrm{a}}$ & $\mathrm{SD}$ & $95 \% \mathrm{Cl}$ & P-value & Mean $^{\mathrm{a}}$ & SD & $95 \% \mathrm{Cl}$ & P-value & Mean $^{\mathrm{a}}$ & $\mathrm{SD}$ & $95 \% \mathrm{Cl}$ & P-value & Mean & $\mathrm{SD}$ & $95 \% \mathrm{Cl}$ & P-value \\
\hline Frontal & 0.2 & 0.8 & $-0.2-0.6$ & 0.249 & 0.2 & 1.9 & $-0.7-1.1$ & 0.699 & -0.8 & 4.5 & $-3.0-1.3$ & 0.423 & -0.3 & 2.7 & $-1.6-1.0$ & 0.644 \\
\hline Temporal & 0.1 & 0.4 & $-0.1-0.3$ & 0.171 & 0.5 & 1.8 & $-0.4--1.4$ & 0.224 & -2.0 & 8.0 & $-5.9-1.9$ & 0.296 & -0.1 & 1.7 & $-0.9-0.7$ & 0.724 \\
\hline Centroparietal & 0.5 & 0.6 & $0.2-0.8$ & 0.004 & 0.7 & 2.9 & $-0.7-2.1$ & 0.298 & 1.3 & 11.2 & $-4.1-6.8$ & 0.611 & 0.0 & 4.8 & $-2.3-2.4$ & 0.982 \\
\hline Occipital & 0.3 & 1.1 & $-0.3-0.8$ & 0.288 & 1.5 & 4.0 & $-0.4-3.4$ & 0.123 & 0.8 & 27.7 & $-12.5-14.2$ & 20.898 & 0.5 & 2.5 & $-0.7-1.7$ & 0.395 \\
\hline
\end{tabular}

a Mean difference,
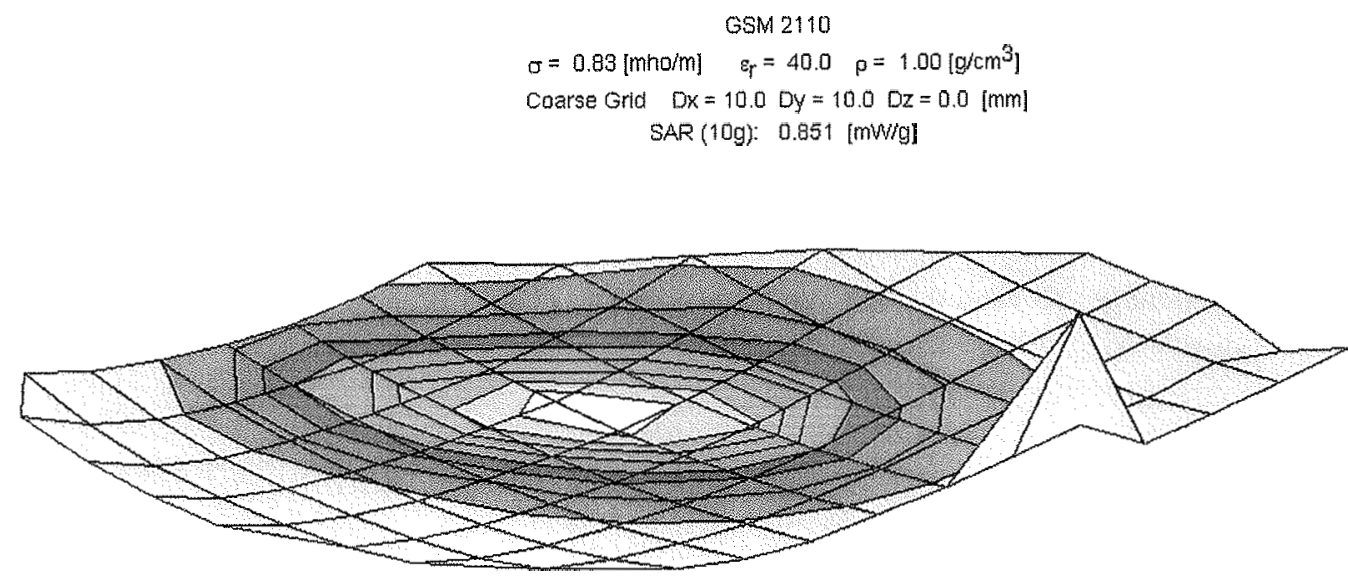

GSM 2110

$\sigma=0.83[\mathrm{mho} / \mathrm{m}] \quad \varepsilon_{\mathrm{r}}=40.0 \quad \rho=1.00\left[\mathrm{~g} / \mathrm{cm}^{3}\right]$ Coarse Grdd $D x=10.0 \quad D y=10.0 \quad 0 z=0.0[\mathrm{~mm}]$ SAR (10g): $0.818[\mathrm{~mW} / \mathrm{g}]$

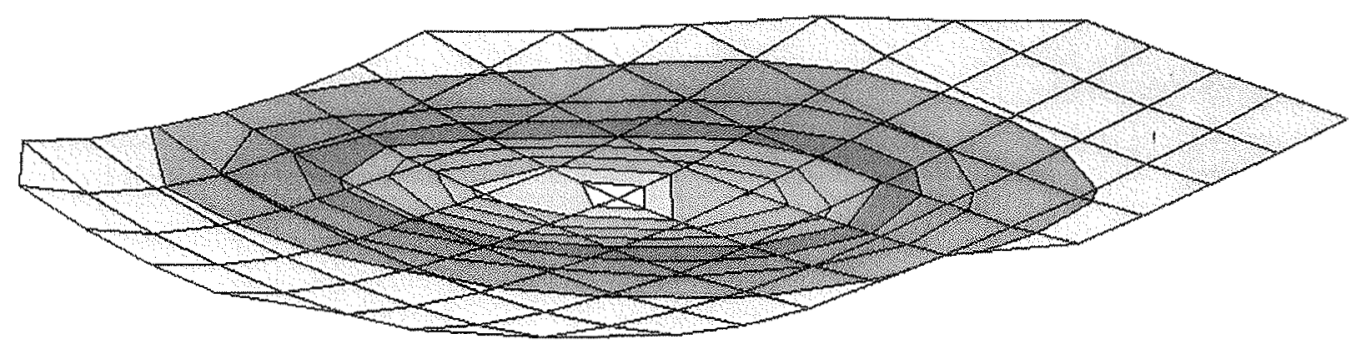

Figure 7. Specific absorption rates (SAR) measured with (a) and without (b) electroencephalographic electrodes inside the phantom head.

different brain sections. If vigilance decreased, the test person was "awakened" by speaking a few words to him or her.

Whether the EEG-recording system acted as a Faraday cage around the subjects' heads was assessed by attaching the EEG electrodes to a "phantom" head. Electric fields inside the liquid head phantom were measured with and without the electrodes by using a scanning system consisting of an electric field probe connected to a robot with data acquisition electronics (figure 2 , see $\mathrm{p}$ 89). The local power absorbed in the phantom was determined by calculating the specific absorption rate (SAR) by the following equation:

$$
\mathrm{SAR}=\sigma \mathrm{E}^{2} / \rho(\mathrm{W} / \mathrm{kg})
$$

(equation 1)

where $\sigma$ is the conductivity, $\rho$ is the density of the averaging mass, and $E$ is the electric field strength. 
various electroencephalographic parameters in the centroparietal region. The exposure source was an NMT phone (900 MHz). (NMT=

\begin{tabular}{|c|c|c|c|c|c|c|c|c|c|c|c|c|c|c|c|c|c|c|c|}
\hline & & & & & & & & & & & & & & & & \multirow{2}{*}{\multicolumn{4}{|c|}{$\begin{array}{l}\text { Mean frequency }\left(\mathrm{mV}^{2}\right) \\
\quad(1.5-25 \mathrm{~Hz})\end{array}$}} \\
\hline \multicolumn{16}{|c|}{ Relative power (\%) } & & & & \\
\hline \multicolumn{4}{|c|}{ Delta } & \multicolumn{4}{|c|}{ Theta } & \multicolumn{4}{|c|}{ Alpha } & \multicolumn{4}{|c|}{ Beta } & \multirow[b]{2}{*}{ Mean $^{\mathrm{a}}$} & \multirow[b]{2}{*}{ SD } & \multirow[b]{2}{*}{$95 \% \mathrm{Cl}$} & \multirow[b]{2}{*}{ P-value } \\
\hline Mean & a $\mathrm{SD}$ & $95 \% \mathrm{Cl}$ & P-value & Mean $^{\mathrm{a}}$ & $S D$ & $95 \% \mathrm{Cl}$ & P-value & Mean $^{\mathrm{a}}$ & $\mathrm{SD}$ & $95 \% \mathrm{Cl}$ & P-value & Mean $^{\mathrm{a}}$ & $S D$ & $95 \% \mathrm{Cl}$ & P-value & & & & \\
\hline 1 & 4 & $-1-3$ & 0.345 & 0 & 4 & $-2-2$ & 0.844 & -1 & 6 & $-4-2$ & 0.588 & 0 & 7 & $-4-3$ & 0.916 & -0.1 & 0.9 & $-0.5-0.4$ & 0.795 \\
\hline 0 & 2 & $-1-1$ & 0.442 & 1 & 3 & $-1-2$ & 0.397 & -1 & 5 & $-3-1$ & 0.354 & 0 & 5 & $-2-2$ & 0.971 & 0.0 & 0.6 & $-0.3-0.3$ & 0.910 \\
\hline 1 & 3 & $-1-2$ & 0.360 & 0 & 3 & $-2-1$ & 0.834 & 0 & 8 & $-4-4$ & 0.957 & 0 & 6 & $-3-3$ & 0.797 & 0.0 & 0.7 & $-0.4-0.3$ & 0.902 \\
\hline 0 & 3 & $-1-2$ & 0.670 & 1 & 3 & $0-2$ & 0.170 & -2 & 7 & $-5-2$ & 0.366 & 0 & 4 & $-2-2$ & 0.674 & 0.0 & 0.5 & $-0.3-0.2$ & 0.843 \\
\hline
\end{tabular}

\section{Data analysis}

The quantitative EEG analyses were made within the $1.5-25 \mathrm{~Hz}$ frequency band using the Neurometrics program of the Cadwell RDC-32 equipment. The analyzed frequency bands were the delta $(1.5-3.5 \mathrm{~Hz})$, theta $(3.5-7.5 \mathrm{~Hz})$, alpha $(7.5-12.5 \mathrm{~Hz})$, and beta $(12.5-$ $25.0 \mathrm{~Hz}$ ) bands. In the monopolar montages (A1A2 as reference), the absolute and relative powers were analyzed in the aforementioned bands for all 21 channels. The mean frequencies within the whole frequency band $(1.5-25 \mathrm{~Hz})$ were determined for each channel. The number of variables was reduced by calculating the regional means from the original variables for 4 regions, which were the frontal region (Fp1, Fp2, Fpz, F3, F4, $\mathrm{Fz}$ ), the temporal region (T3, T4, T5, T6), the centroparietal region $(\mathrm{C} 3, \mathrm{C} 4, \mathrm{Cz}, \mathrm{P} 3, \mathrm{P} 4, \mathrm{Pz})$, and the occipital region $(\mathrm{O} 1, \mathrm{O} 2, \mathrm{Oz})$. After the calculations the number of the EEG variables was 36 (16 for the absolute power, 16 for the relative power, and 4 for the mean frequencies).

The statistical analysis was based on comparisons of the EEG changes during the actual exposure with the changes during the sham exposure. The statistical significance of the differences of the analyzed results between the actual and sham exposure registrations were tested with the Student's paired t-test. Differences were considered statistically significant at $\mathrm{P}<0.01$.

\section{Results}

The statistical analysis was made by comparing the EEG changes of each test person during actual exposure with his or her own EEG changes during the sham exposure. The mean difference of the absolute powers $\left(\mu V^{2}\right)$ in the 4 brain regions with 5 cellular phone models are illustrated in figures $3-6$ (see p 89). The differences in the EEG parameters recorded during the sham and actual exposures were extremely small. The statistical calculations indicated that only one significant $\mathrm{P}$-value $(\mathrm{P}=0.004)$ was obtained in the t-tests. The difference occurred for the absolute power of the delta band during exposure to one of the NMT phones. In this case, the absolute power in the centroparietal region was, on the average, $0.5 \mu \mathrm{V}^{2}$ greater than during the sham exposure. The $95 \%$ confidence interval $(95 \% \mathrm{CI})$ of the difference was $0.2-0.8$ $\mu \mathrm{V}^{2}$ (table 1). However, the difference in the relative power between the actual and sham exposure in the same band and the same region was only $1 \%(95 \% \mathrm{CI}-1-$ $2 \%$ ).

The results of the test concerning the possible Faraday effect of the EEG electrodes indicated that the wiring around the head did not cause any attenuation in the absorption of RF fields. Figure 7 indicates that the measured SAR values were nearly the same with and without the electrodes.

\section{Discussion}

The effect of RF electromagnetic fields emitted from a cellular phone on the awake EEG was studied in a single-blind, placebo-controlled experiment. The differences between the sham and actual exposures were extremely small, only one statistically significant difference being observed. The observed difference was probably caused by statistical chance in relation to the multitesting situation (180 t-tests calculated). If the difference had been real, the same kind of difference should have been observed for the relative power, which was not the case.

The assessment of the effects of RF exposure on the EEG was made by comparing each person's EEG recordings to his or her own sham recording. Because the EEG spectra of healthy adults are repeatable after a period of about a year and the statistical method used is very sensitive, even the smallest differences, if there were any, should have been detected. As regards exposure times, cellular phone calls are normally short, and the duration of an experimental trial was adequate for detecting acute effects if they had existed.

In summary, the results of our study indicate that RF fields generated by cellular phones have no adverse 
effects on human brain activity. On the other hand, these experiments cannot be regarded as definite data concerning all possible abnormal changes in human brain function during the use of cellular phones. The findings of EEG studies refer only to a limited portion of cerebral functions, and they cannot be extended to other physiological parameters. In addition, the study population consisted of healthy, rather young subjects. In terms of moresensitive subpopulations, for example, children and elderly people, possible effects may occur at lower SAR levels. Therefore, additional studies are needed, specifically with a careful determination of the exposure parameters and set-ups.

\section{Acknowledgments}

We would like to express our thanks to Ms Riitta Velin for recording the EEG spectra and Mr Patrick von Nandelstadh for helping in the exposure arrangements. This study was a part of the Finnish COST 244. Project, funded mainly by the Technical Development Centre of Finland (TEKES).

\section{References}

1. Anderson V, Joyner KH. Specific absorption rate levels measured in a phantom head exposed to radio frequency transmis- sions from analog hand-held mobile phones. Bioelectromagnetics 1995;16:60-9.

2. Balzano Q, Garay O, Steel FR. Energy deposition in simulated human operators of $800-\mathrm{MHz}$ portable transmitters. IEEE Trans Vehicular Techn 1978;VT-27:174-181.

3. Spiegel J. The thermal response of a human in the near-zone of a resonant thin-wire antenna. IEEE Trans Microwave Theory Techn 1982;MTT-30:177-185.

4. Bell G, Marino A, Chesson A, Struve F. Electrical states in the rabbit brain can be altered by light and electromagnetic fields. Brain Res 1992;570:307-15.

5. Lyskov EB, Juutilainen J, Jousmäki V, Partanen J, Medvedev $\mathrm{S}$, Hänninen $\mathrm{O}$. Effects of $45-\mathrm{Hz}$ magnetic fields on the functional state of the human brain. Bioelectromagnetics 1993;14:87-95.

6. Lyskov E, Juutilainen J, Jousmäki $V$, Hänninen $O$, Medvedev $S$, Partanen J. Influence of short-term exposure of magnetic field on the bioelectrical processes of the brain and performance. Int J Psychophysiol 1993;14:227-31.

7. Lyskov E, Juutilainen J, Koivisto E, Yavich L, Riekkinen P. Effects of 45-Hz magnetic fields on cortical EEG spectrum in rats. Electro Magnetobiol 1993;12:179-185.

8. Reiser H, Dimpfel W, Schober F. The influence of electromagnetic fields on human brain activity. Eur J Med Res $1995 ; 1: 27-32$.

9. Röschke J, Mann K. No short-term effects of digital mobile radio telephone on the awake human electroencephalogram. Bioelectromagnetics 1997; 18:172-6.

10. Freude G, Ullsperger P, Eggert S, Ruppe I. Effects of microwaves emitted by cellular phones on human slow brain potentials. Bioelectromagnetics 1998;19:384-7.

Received for publication: 14 April 1999 